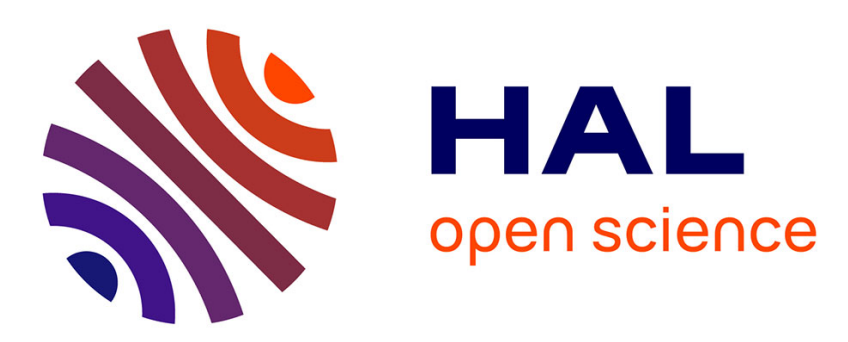

\title{
Une approche pour la configuration cohérente de produit et de gamme de production
}

Michel Aldanondo, Élise Vareilles, Yosra Lahmar, Claude Baron, Guillaume Moynard

\section{- To cite this version:}

Michel Aldanondo, Élise Vareilles, Yosra Lahmar, Claude Baron, Guillaume Moynard. Une approche pour la configuration cohérente de produit et de gamme de production. Journal Européen des Systèmes Automatisés (JESA), 2007, 41 (5), pp.568-584. hal-01847758

\section{HAL Id: hal-01847758 \\ https://hal.science/hal-01847758}

Submitted on 28 Nov 2019

HAL is a multi-disciplinary open access archive for the deposit and dissemination of scientific research documents, whether they are published or not. The documents may come from teaching and research institutions in France or abroad, or from public or private research centers.
L'archive ouverte pluridisciplinaire HAL, est destinée au dépôt et à la diffusion de documents scientifiques de niveau recherche, publiés ou non, émanant des établissements d'enseignement et de recherche français ou étrangers, des laboratoires publics ou privés. 


\title{
Une approche pour la configuration cohérente de produit et de gamme de production
}

\author{
Michel Aldanondo* — Elise Vareilles* — Yosra Lahmar* \\ Claude Baron** — Guillaume Moynard*** \\ * Centre Génie Industriel - Ecole des Mines d'Albi-Carmaux, F-81000 Albi \\ \{aldanondo,vareilles, lahmar\}@enstimac.fr \\ ** Laboratoire d'Etudes des Systèmes Informatiques et Automatiques \\ Institut National des Sciences Appliquées, F-31077 Toulouse \\ claude.baron@insa-toulouse.fr \\ *** Lapeyre informatique, F-44800 Saint Herblain \\ guillaume.moynard@lapeyre.fr
}

\begin{abstract}
RÉSUMÉ. Les techniques de personnalisation ou de configuration de produits ne considèrent pas le plus souvent la définition des gammes de production. La production dépendant fondamentalement du produit configuré, cet article vise à montrer comment les approches par contraintes, exploitées pour la configuration de produit, peuvent être utilisées en parfaite complémentarité pour configurer les gammes de production. Après une brève introduction, une première partie aborde les principes de base de la configuration de produit. Ensuite, une approche permettant la configuration de gamme de production prenant en compte la configuration de produit est proposée. Un exemple détaillé illustre tous les éléments proposés tout au long de la communication.

ABSTRACT. Product configuration or customisation techniques do not consider, most of the time the definition of the relevant process routings. As manufacturing and assembling strongly depend on product definition, the goal of this paper is to show how constraint based configuration techniques can be smoothly extended in order to configure process routings. Following a short introduction, a first section recalls configuration basics. Then an approach allowing production process configuration with respect to the configured product is proposed. A detailed example illustrates the propositions all along the paper.

MOTS-CLÉS: configuration de produit, configuration de gamme de production, phases, gammes, ressources, problème de satisfaction de contraintes.

KEYWORDS: product configuration, process configuration, operations, routings, constraints satisfaction problem.
\end{abstract}




\section{Introduction}

La diversité des produits industriels mis sur le marché augmentant sans cesse, de nombreuses entreprises industrielles sont confrontées à l'optimisation de cette diversité. C'est-à-dire rechercher un compromis entre les coûts de gestion des références produit et les coûts de suréquipement des références produit. Une solution, fréquemment rencontrée qui permet d'éviter cette recherche délicate de compromis, est de recourir aux techniques de configuration de produit permettant de satisfaire au plus juste les besoins clients de manière spécifique. Pour les entreprises manufacturières, ce problème de configuration ou de personnalisation de l'offre se rencontre fondamentalement pour la définition des produits ou familles de produits mais également pour la définition de leurs procédés ou gammes de production (fabrication, assemblage, packaging...).

Si de nombreux travaux se sont intéressés à la configuration de produit et aux progiciels aidant le processus de configuration de produit, voir les numéros sur la configuration (IEEE, 1998, AIEDAM, 1998 et AIEDAM, 2003), à notre connaissance, la configuration des gammes de production n'a pas été l'objet de travaux spécifiques. De nombreux auteurs, entre autres (Mittal et al., 1989; Sabin et al., 1996; Aldanondo et al., 2003), ayant montré que la configuration de produit pouvait se formaliser comme un problème de satisfaction de contraintes le but de cet article est de combler une lacune dans le domaine en montrant comment les techniques de satisfaction de contraintes peuvent être utilisées pour configurer de manière cohérente le produit et sa gamme de production.

La première section présente ce que recouvre la notion de configuration de produit et comment les approches par contraintes permettent d'aider le processus de configuration, c'est-à-dire comment la configuration de produit peut se formaliser comme un problème de satisfaction de contraintes. La seconde section, en exploitant les éléments décrits dans la première partie, propose des éléments permettant de configurer les gammes de production en prenant en compte le produit configuré. Nous nous intéresserons principalement à l'aspect modélisation des problèmes plutôt qu'à sa résolution qui fait appel à des techniques classiques de propagation de contraintes et de recherche de solutions. Les éléments proposés correspondant avant tout à des recommandations de modélisation, un exemple simple mais étudié de manière complète illustre toutes les propositions afin de permettre une compréhension détaillée des problématiques et des solutions proposées.

\section{Configuration de produit}

La première sous-section rappelle dans un premier temps la définition de la configuration de produit. Il est ensuite montré comment ce problème peut être considéré comme un problème de satisfaction de contraintes. En fin de première partie, une contrainte particulière correspondant à un raccourci de modélisation 
permettant une représentation structurée des modèles nécessaire à nos

propositions est définie.

\subsection{Configuration de produit et configurateur}

En étudiant les travaux variés sur la configuration de produit, il apparaît que les éléments communs permettant de définir la configuration de produit sont :

- hypothèse : un produit est un ensemble de composants.

Etant donné :

- un modèle générique d'un produit configurable capable de représenter une famille de produits avec toutes ses possibilités de variantes et d'options, qui met en œuvre :

- des groupes de composants,

- des propriétés de composant ou de produit,

- des contraintes qui limitent les possibilités de combinaison de composants et de valeurs des propriétés,

- un ensemble de besoins client, où chaque besoin s'exprime sous la forme d'une sélection de composant dans un groupe ou d'un choix de valeur pour une propriété pouvant éventuellement correspondre à une caractéristique fonctionnelle.

Configurer peut se définir comme «trouver un ou tous les ensembles de composants qui satisfont les contraintes du modèle générique et les besoins client ».

Ces éléments de définition peuvent être consultés dans (Mittal et al., 1989; Soininen et al., 1998 ; Aldanondo et al., 2003). Il est important de noter que selon cette définition, le résultat du processus de configuration est un ensemble de composants correspondant à la nomenclature du produit configuré.

Un progiciel de configuration ou configurateur est un progiciel qui aide le déroulement $\mathrm{du}$ processus de configuration. Il est composé d'une base de connaissances contenant le modèle générique du produit configurable et d'un ensemble d'outils d'assistance à la configuration. La base de connaissances est le plus souvent une base de données relationnelles qui permet de stocker le modèle générique produit. Ce modèle générique rassemblant toute la connaissance relative à la diversité produit se dénomme, souvent par abus de langage, base de connaissances ou connaissances du domaine. Ces connaissances, nécessaires à l'élaboration des modèles, sont obtenues auprès des experts produits des entreprises dans les services marketing, commerciaux, études, industrialisation et production. Les outils d'assistance aident l'utilisateur à instancier le modèle générique suivant les besoins clients, c'est-à-dire à choisir des composants et des valeurs des propriétés tout en respectant les contraintes du modèle. Le rôle fondamental de ces outils est de garantir que le produit configuré est cohérent (c'est-à-dire qu'il respecte les contraintes du modèle et les besoins utilisateur) pendant et à la fin du processus de 
configuration. Des éléments détaillés concernant les fonctionnalités et constituant des progiciels de configuration peuvent être consultés dans (Sabin et al., 1998 ; Aldanondo et al., 2001).

L'exemple illustrant cet article est une version simplifiée d'une armoire de rangement proposée dans (Moynard, 2003) et représentée en figure 1. Quatre groupes de composants existent : grand casier de deux hauteurs : $72 \mathrm{~cm}$ ou $216 \mathrm{~cm}$ (BC), casier haut de hauteur $144 \mathrm{~cm}$ (HC), casier bas de hauteur $72 \mathrm{~cm}$ (LC) et étagères pouvant aller dans le grand casier (RO). Tous les composants ont deux finitions : peinture (P) ou vernis (W). De ce fait, les groupes de composants se définissent de la manière suivante :
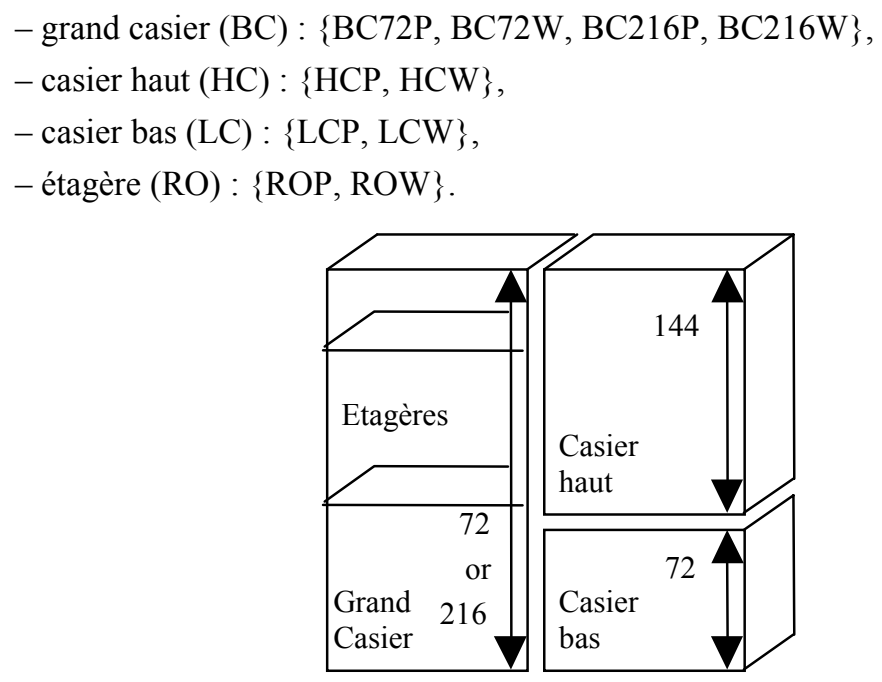

Figure 1. Exemple de produit configurable

Les possibilités d'assemblage sont limitées par les contraintes suivantes :

- tous les composants d'un produit configuré doivent avoir la même finition,

- le grand casier est présent dans tous les produits configurés, les casiers haut et bas sont présents suivant le besoin,

- le casier haut peut être présent dans le produit configuré uniquement si le casier bas est présent et si la hauteur du grand casier est de $216 \mathrm{~cm}$,

- le nombre d'étagères dans le grand casier est de 1 ou 2 si la hauteur du grand casier est de $72 \mathrm{~cm}$ et de 1, 2, 3 ou 4 si la hauteur du grand casier est de $216 \mathrm{~cm}$.

Les propriétés du produit sont alors :

- hauteur du grand casier $(\mathrm{BCH}):\{72,216\}$,

- finition du produit (PFH) : \{Wood, Painted $\}$, 
- présence du casier bas (LCX) : \{Yes, No\},

- présence du casier haut $(\mathrm{HCX}):\{$ Yes, No\},

- nombre d'étagères (QRO) : $\{1,2,3,4\}$.

\subsection{Configuration et approches par contraintes}

Les problèmes de satisfaction de contraintes (CSP) définis par (Montanari, 1974) comme un triplet $\{\mathrm{X}, \mathrm{D}, \mathrm{C}\}$, où $\mathrm{X}$ est un ensemble de variables, $\mathrm{D}$ un ensemble de domaines (un pour chaque variable) et $\mathrm{C}$ un ensemble de contraintes de compatibilité (définissant les combinaisons de valeurs de variables autorisées ou exclues), peuvent représenter partiellement la combinatoire des modèles génériques des produits configurables. Chaque groupe de composants et chaque propriété de produit sont associés à une variable. Chaque composant et chaque valeur de propriété sont associés à une valeur de variable. Les contraintes représentent les combinaisons, autorisées ou exclues (lignes respectivement pleines ou pointillées en figure 2), de composants et de valeurs de propriétés. L'extension des CSP proposée par (Mittal et al., 1990), les CSP dynamiques ou DCSP (fréquemment dénommés conditionnels) ajoutent les notions de :

- variables initialement actives : toujours présentes et nécessaires pour définir tout produit configuré, ou inactives : éventuellement présentes pour définir certains produits configurés,

- contraintes d'activation : contraintes qui permettent de déclencher l'existence ou activation d'une variable afin de définir certains produits configurés, où une valeur « $\mathrm{X}$ » de la variable $\mathrm{X}$ implique l'existence de la variable $\mathrm{Y}$, notée : $(\mathrm{X}=$ « $\mathrm{X} ») \rightarrow \mathrm{Y}$

Cette extension permet de modéliser à l'aide de variables la présence de composants ou de propriétés dans le produit. Avec ces éléments, l'exemple de produit configurable de la section précédente peut se modéliser, comme représenté en figure 2, en faisant apparaître :

- 4 variables correspondant aux 4 groupes de composants : BC, RO, LC, HC, avec $\mathrm{BC}$ et $\mathrm{RO}$ initialement actives, $\mathrm{LC}$ et $\mathrm{HC}$ initialement inactives,

- 5 variables correspondant aux 5 propriétés : BCH, PFH, LCX, HCX, QRO, toutes ces variables étant initialement actives,

-8 contraintes de compatibilité entre les variables suivantes :

- 4 contraintes montrant les sélections possibles de composants suivant la propriété de finition : (PFH, BC), (PFH, RO), (PFH, LC), (PFH, HC),

- 1 contrainte excluant les grands nombres d'étagères pour une hauteur faible du grand casier (BCH, QRO),

- 1 contrainte montrant les sélections de grand casier suivant la hauteur du grand casier $(\mathrm{BCH}, \mathrm{BC})$, 
- 1 contrainte excluant la présence du casier haut suivant la hauteur du grand casier (BCH, HCX),

- 1 contrainte excluant la présence du casier haut si le casier bas est absent (LCX, HCX),

- 2 contraintes d'activation contrôlant l'existence des groupes optionnels de composants : casier bas (LC) : $(\mathrm{LCX}=\ll$ Yes $») \rightarrow$ LC et casier haut $(\mathrm{HC}):(\mathrm{HCX}=$ « Yes ») $\rightarrow \mathrm{HC}$.

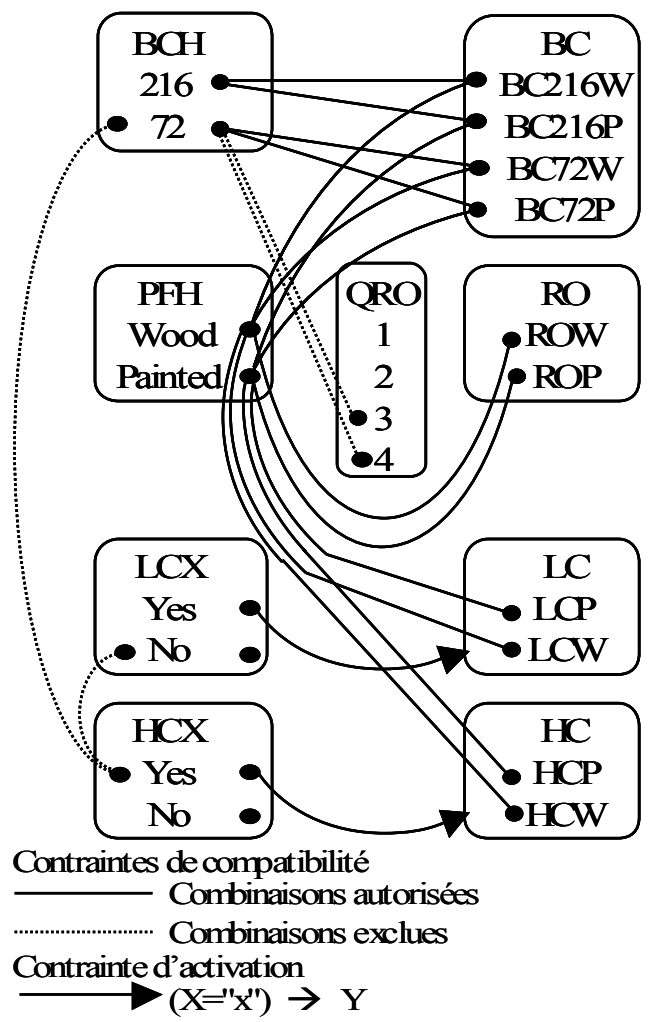

Figure 2. Modèle de l'exemple

Les variables de la partie gauche et du centre du modèle de la figure 2 permettent à l'utilisateur de saisir son besoin (BCH, PFH, LCX, HCX et QRO). Les variables de la partie droite et du centre du modèle correspondent aux composants apparaissant dans la nomenclature du produit configuré (BC, RO, QRO, LC et HC). Ce modèle représente une combinatoire de 32 solutions. Un exemple de besoin: $\mathrm{BCH}=$ «216», $\mathrm{PFH}=$ « Wood », $\mathrm{LCX}=$ «Yes », $\mathrm{HCX}=$ «Yes » et $\mathrm{QRO}=\ll 2 »$, fournit la liste de composants ou nomenclature : $\{\mathrm{BC} 216 \mathrm{~W}, \mathrm{LCW}, \mathrm{HCW}, 2 \mathrm{ROW}\}$. 
L'aide au processus de configuration est fournie par diff érents mécanismes de résolution et de propagation de contraintes comme ceux proposés ou discutés par (Mittal et al., 1990 ; Soininen et al., 1999) ou (Gelle et al., 2003).

\subsection{Représentation structurée et raccourci de modélisation}

Il est clair que le modèle produit proposé n'est pas structuré ou hiérarchisé, signifiant qu'un produit est simplement un ensemble de composants représenté par une nomenclature de type «râteau ». Le plus souvent, il est nécessaire de pouvoir représenter plusieurs niveaux de sous-ensembles et donc d'avoir une forme de représentation arborescente ou hiérarchique du produit. De même lors de la représentation des gammes de production, il sera nécessaire d'avoir une représentation structurée pour pouvoir représenter la décomposition de la gamme de production en opérations et phases.

Faire face à ce besoin nécessite une extension du formalisme CSP. Seuls quelques auteurs se sont intéressés à ce problème. Les « Composite CSP » introduits par (Sabin et al., 1996) considèrent qu'une valeur d'une variable peut correspondre elle-même à un CSP. Lorsque cette valeur est sélectionnée, la variable est alors remplacée par le CSP correspondant et permet une forme de modélisation hiérarchique des problèmes. Les «State CSP », proposés par (Véron et al., 2000) associant à chaque variable une variable d'état, permettent de même d'encoder les modèles avec une approche hiérarchique.

L'idée préconisée dans les «Composite CSP » étant très proche de notre besoin de structure, nous proposons de l'adapter à l'aide du formalisme DCSP utilisé précédemment. L'idée est de proposer un raccourci de notation basé sur les contraintes d'activation de variables des DCSP permettant l'activation d'un sousmodèle DCSP. Plus formellement, un sous-modèle DCSP, SM, étant défini par :

- un ensemble de variables V1 initialement actives,

- un ensemble de variables V2 initialement inactives,

- un ensemble de contraintes de compatibilité,

- un ensemble de contraintes d'activation conditionnant l'existence des variables initialement inactives :

$\forall \mathrm{v} 2 \in \mathrm{V} 2,(\mathrm{vk}=$ Cste-k) $\rightarrow \mathrm{v} 2$, with $\mathrm{vk} \in \mathrm{V} 1 \cup \mathrm{V} 2$.

Lorsque ce sous-modèle SM est inclus dans un modèle de niveau supérieur et que son existence est conditionnée par une valeur (par exemple « vsm») d'une variable (par exemple $\mathrm{V}-\mathrm{x}$ ) du modèle de niveau supérieur, toutes les variables du sousmodèle SM doivent être contraintes de la manière suivante :

- chaque variable $\mathrm{v} 1 \in \mathrm{V} 1$ est alors initialement localement active et globalement inactive et est contrainte par : 


$$
\forall \mathrm{v} 1 \in \mathrm{V} 1,(\mathrm{~V}-\mathrm{x}=\ll \mathrm{vsm} ») \rightarrow \mathrm{v} 1
$$

- les contraintes d'activation des variables v2 $\in \mathrm{V} 2$ sont cumulées avec la contrainte d'activation de sous-modèle de la manière suivante :

$$
\forall \mathrm{v} 2 \mathrm{~V} 2,(\mathrm{~V}-\mathrm{x}=\ll \mathrm{vsm} ») \wedge(\mathrm{vk}=\mathrm{Cste}-\mathrm{k}) \rightarrow \mathrm{v} 2
$$

Le formalisme DCSP considérant qu'une contrainte est active uniquement lorsque toutes les variables qu'elle lie sont actives, les contraintes liant des variables du sous-modèle ont en conséquence une activation conditionnée. Nous dénommons ce raccourci de notation «activation de sous-modèle» et le notons ( $\mathrm{V}-\mathrm{x}=$ « vsm ») $\rightarrow \mathrm{SM}$, où SM est inactif et contient un ensemble de variables localement actives ou inactives.

L'intérêt de ce raccourci est principalement représentatif, car il permet lors de la modélisation d'éviter de définir des prémisses compliqués pour les contraintes d'activation. Les modèles obtenus pouvant se traduire sans problème dans le formalisme DCSP, les algorithmes de résolution et de filtrage propres aux DCSP peuvent être utilisés.

L'exemple de la figure 2 est à nouveau considéré, mais nous faisons maintenant l'hypothèse que le produit est composé de deux sous-ensembles : le premier BC-RO comprend le grand casier et les étagères, tandis que le second $\mathrm{HC}-\mathrm{LC}$ rassemble le casier bas et le casier haut. Chaque sous-ensemble constitue alors individuellement un produit configurable qui peut être utilisé dans différents produits de niveau supérieur.

La figure 3 représente le modèle de produit avec l'approche structurée proposée. Le modèle de niveau haut ne comprend plus que les variables HC-LC-X et BC-RO$\mathrm{X}$ activant les sous-modèles. Un sous-modèle est associé à chaque sous-ensemble et conditionné par une contrainte d'activation de sous-modèle :

$$
\begin{aligned}
& -(\mathrm{HC}-\mathrm{LC}-\mathrm{X}=\langle\text { Yes } ») \rightarrow \text { HC-LC, } \\
& -(\mathrm{BC}-\mathrm{RO}-\mathrm{X}=\langle\text { Yes } ») \rightarrow \mathrm{BC}-\mathrm{RO} .
\end{aligned}
$$

Le sous-modèle HC-LC rassemble les variables PFH, HCX, LC et HC et le sousmodèle $\mathrm{BC}-\mathrm{RO}$ rassemble les variables $\mathrm{BCH}, \mathrm{PFH}, \mathrm{QRO}, \mathrm{BC}$ et $\mathrm{RO}$. La variable $\mathrm{PFH}$ est dupliquée dans les deux sous-modèles afin de permettre la configuration autonome des deux sous-ensembles.

L'espace des configurations possibles est identique à celui du modèle de la figure 2. L'exemple de besoin décrit en fin de section 2.2 devient avec ce modèle :

- au niveau produit : HC-LC-X $=$ «Yes », BC-RO-X $=$ «Yes »,

- pour le sous-ensemble $\mathrm{BC}-\mathrm{RO}: \mathrm{BCH}=\langle 216 », \mathrm{PFH}=\langle$ Wood $», \mathrm{QRO}=\langle 2 »$,

- pour le sous-ensemble HC-LC : HCX $=«$ Yes $», \mathrm{PFH}=«$ Wood $»$. 
Le résultat en termes de nomenclature est alors :

- au niveau produit : 1 sous-ensemble HC-LC, 1 sous-ensemble BC-RO,

- pour le sous-ensemble BC-RO : 1 BC216W, 2 ROW,

- pour le sous-ensemble HC-LC : $1 \mathrm{LCW}, 1 \mathrm{HCW}$.

Les éléments concernant la configuration de la nomenclature du produit étant en place, la section suivante a pour but de montrer comment la gamme de production peut être elle même configurée en cohérence avec la nomenclature du produit configuré.

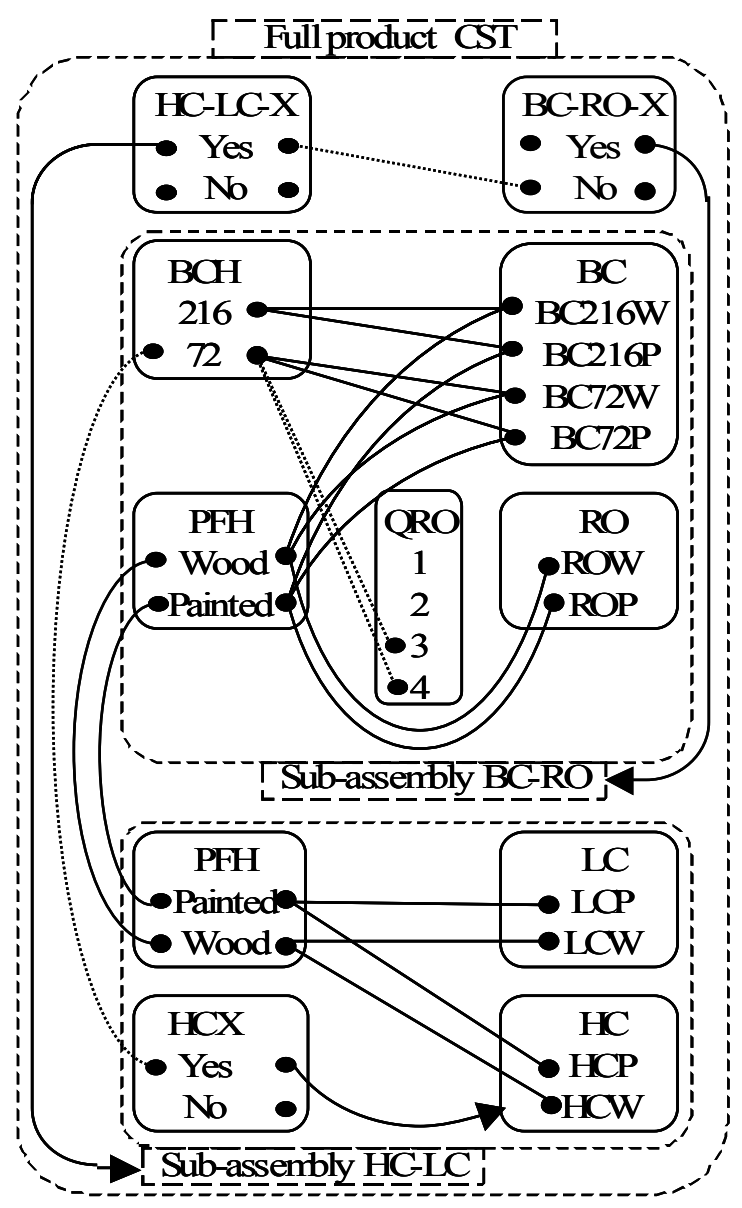

Figure 3. Modèle avec approche structurée 


\section{Configuration de gamme de production}

Cette section concerne la configuration de la gamme de production en cohérence avec la configuration de produit. Le formalisme CSP et ses extensions introduits en partie 1 sont utilisés sans modification. La section 3.1 décrit ce que nous entendons par configuration de gamme de production et introduit les notions de gamme, d'opération et de phase. Les sections suivantes montrent comment la configuration de gamme (section 3.2) et la configuration de phase (section 3.3) peuvent être considérées comme des problèmes de satisfaction de contraintes. Dans chaque section les liens avec la configuration de produit sont explicités.

\subsection{Configuration de gamme de production}

Très peu d'auteurs se sont intéressés à la configuration de la gamme de production. Il est cependant possible de citer (Hvam et al., 2002) qui ont étudié le processus de configuration dans sa globalité en faisant apparaître le cycle de vie du produit configurable, comprenant entre autres la configuration de la gamme de production. Ces travaux, orientés analyse par processus, ne fournissent malheureusement pas d'élément de modélisation de ce problème.

La gamme de production représente l'enchaînement des activités nécessaires à la production d'un produit. Une gamme de production est un ensemble d'opérations reliées par des contraintes d'antériorité représentant un ordre total. Chaque opération est associée à une ou plusieurs phases. Chaque phase est définie par un ensemble de ressources requises qui permettent de réaliser la phase, chaque réquisition de ressource est caractérisée par une quantité.

Cette représentation simple ne mettant en œuvre qu'un seul niveau de décomposition gamme/phase a été choisi pour clarifier le message. Il est bien sûr possible de considérer des décompositions multiniveaux utilisant par exemple une décomposition macrogamme/gamme/macrophase/phase/instruction. Cependant les ressources apparaîtront toujours au plus bas niveau de décomposition. En conséquence nous ne parlerons que de modèles de gamme d'opération et de phase générique.

\subsection{Configuration de gamme}

Une gamme étant composée d'opérations reliées par des contraintes d'antériorité, un sous-modèle sera associé à chaque opération (noté Op). L'ensemble de ces sousmodèles constituera le modèle de la gamme.

Les contraintes d'antériorité entre opérations sont modélisées à l'aide du concept de «port» (noté Po) proposé par (Mittal et al., 1989). Cette notion souvent utilisée en configuration de produit permet de représenter le fait que certains composants 
doivent être connectés en faisant appel à des «variables port» indiquant les possibilités de connexion à des ports d'autres composants. Nous proposons d'utiliser cette idée et d'associer à chaque opération une ou plusieurs variables ports (noté OpPo). Ces variables ports sont de type «précédant», (noté OpPo-) ou de type «suivant» (noté $\mathrm{OpPo}^{+}$) et ont pour valeurs possibles les ports des opérations respectivement précédentes et suivantes envisageables. Une opération qui n'a pas de variable port $\mathrm{OpPo}$ - ou $\mathrm{OpPo}+$ est une opération respectivement de début de gamme ou de fin de gamme. Plusieurs ports de même type associé à une même opération permettent de représenter des convergences (OpPo-) ou des divergences $\left(\mathrm{OpPo}^{+}\right)$ d'opérations. Les contraintes reliant les variables ports matérialisent l'utilisation des ports en indiquant les combinaisons de valeurs des variables ports $\mathrm{OpPo}+\mathrm{OpPo}-\mathrm{de}$ phases différentes pouvant être deux à deux connectées.

Chaque sous-modèle d'opération est donc constitué de la variable opération $(\mathrm{Op})$ dont les valeurs sont des phases et des variables port (OpPo). Les contraintes entre les ports sont associées au modèle de la gamme. Les liens entre les éléments de ce modèle et le modèle du produit générique sont de deux types :

- les sous-modèles de chaque opération peuvent être reliés au modèle du produit générique avec des contraintes d'activation de sous-modèle et permettre de moduler l'existence des opérations en fonction du produit configuré,

- les variables Op peuvent être reliées au modèle du produit générique par des contraintes de compatibilité afin de sélectionner la phase correspondant au produit configuré.

Les liens entre ce modèle et le modèle des phases (section 3.3) seront de même des contraintes d'activation de sous-modèle permettant une fois la phase sélectionnée pour l'opération d'activer le sous-modèle de la phase générique.

Ces éléments de modélisation permettent de représenter, en figure 4, la gamme générique de l'exemple introduit en section 2. La gamme comporte quatre opérations associées chacune à un sous-modèle comprenant les variables suivantes :

- opération de fabrication « Fabr BCRO » du sous-ensemble BC-RO constitué du grand casier et des étagères. Cette opération est toujours présente dans la gamme et est définie par les variables :

- Op-1 : associée à une seule phase « $\mathrm{Ph} 1 »$,

- Op-1-Po+ : port indiquant les ports des deux opérations pouvant succéder : assemblage final des sous-ensemble Op-3-Po1- ou packaging final Op-4-Po1(divergence en «OU »),

- pas de port précédant car Op-1 est une opération de début de gamme,

- opération de fabrication «Fabr HCLC » du sous-ensemble HC-LC constitué des casiers bas et haut. Cette opération a une existence conditionnée par le produit configuré et est définie par les variables : 
- Op-2 : associée à une seule phase « $\mathrm{Ph} 2 »$,

- Op-2-Po+: port indiquant le port de l'unique opération devant succéder : assemblage final des sous-ensembles Op-3-Po2-,

- pas de port précédant car Op-2 est une opération de début de gamme.

- opération d'assemblage finale «Ass final» des deux sous-ensembles. Cette opération a une existence conditionnée par le produit configuré et est définie par les variables :

- Op-3 : associée à une seule phase « $\mathrm{Ph} 3 »$,

- Op-3-Po+: port indiquant le port de l'unique opération devant succéder : packaging final Op-4-Po2-, Op-3-Po1- et Op-3-Po2- : deux ports indiquant le port des deux phases devant respectivement précéder : fabrication de BCRO Op-1-Po+ et fabrication de HCLC Op-2-Po+ (convergence en «ET »).

- opération de packaging final «Pack final» du produit configuré. Cette opération est toujours présente dans la gamme et est définie par les variables :

- Op-4 : associée à deux phases «Ph4-1» et «Ph4-2» suivant le type de finition du produit configuré respectivement « peinte » ou « vernie »,

- pas de port suivant car Op-4 est une opération de fin de gamme,

- Op-4-Po- : port indiquant les ports des deux opérations pouvant précéder : fabrication de BCRO Op-1-Po+ ou assemblage final des sous-ensembles Op-3-Po+ (convergence en « $\mathrm{OU} »$ ).

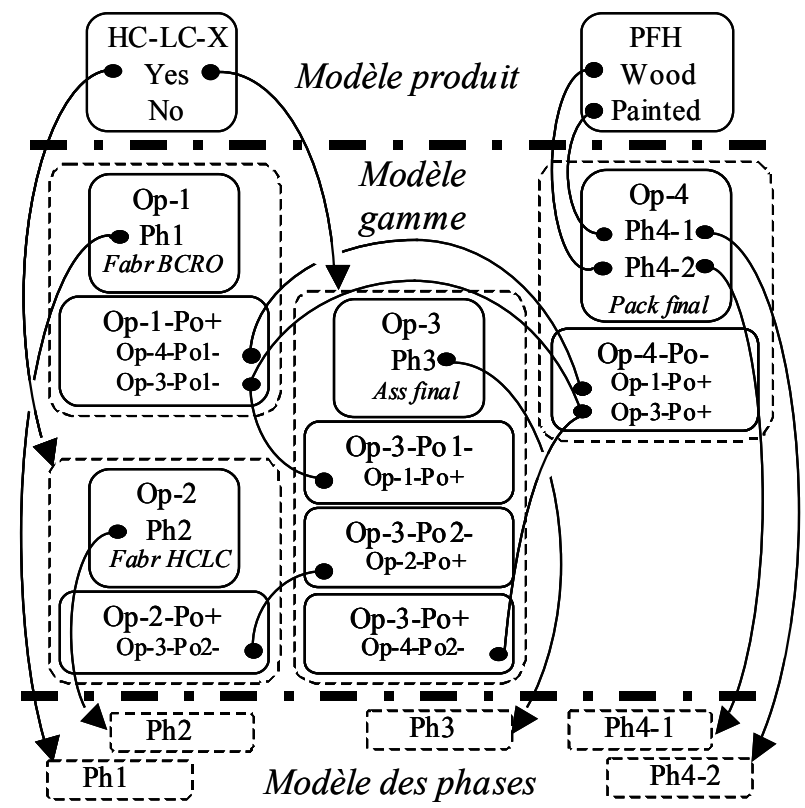

Figure 4. Modèle de la gamme de l'exemple 
Les contraintes entre les valeurs des variables port montrent uniquement les compatibilités de connexion des opérations deux à deux. Deux types de compatibilités peuvent exister. La première est évidente et indique que si une phase $\mathrm{i}$ est suivie par une phase $\mathrm{j}$ alors la phase $\mathrm{j}$ doit être précédée par la phase $\mathrm{i}$, la valeur «Op-j-Po- de la variable port Op-i-Po+ doit être compatible avec la valeur «Op-i$\mathrm{Po}^{+} »$ de la variable port Op-j-Po-, ce type de contrainte se retrouve entre : Op2 et Op3, Op3 et Op4, Op1 et Op4 lorsque Op3 n'existe pas. La seconde est moins évidente et montre que si une phase i est suivie par une phase k' alors la phase $\mathrm{j}$ peut être précédée par une phase k" (les phases k' et k" peuvent correspondre à une même phase). Ce type de contrainte se retrouve entre Op1 et Op4 et stipule que si Op1 et Op3 se suivent alors Op3 et Op4 se suivent (dans ce k'=k"= Op3).

Les éléments de ce modèle de gamme sont reliés au modèle produit de deux manières. D'une part, le sous-ensemble regroupant les caissons bas et haut HCLC étant optionnel, deux contraintes d'activation de sous-ensemble permettent de contrôler l'existence des opérations Op-2 « Fabr HCLC » et Op-3 « Ass final» :

$-($ HC-LC-X $=\ll$ Yes $») \rightarrow$ Op-2,

$-($ HC-LC-X $=«$ Yes $») \rightarrow$ Op-3.

D'autre part, suivant la finition du produit, la phase de packaging est différente, ceci est modélisé avec la contrainte de compatibilité entre les variables PFH et Op-4.

Ce modèle de gamme est également relié aux sous-modèles des phases (section 3.3) par des contraintes d'activation permettant suivant chaque valeur $\left(\mathrm{Ph} \_\mathrm{m}\right)$ des variables opérations $\left(\mathrm{Op} \_\mathrm{n}\right)$ d'activer les sous-modèles correspondant aux phases $\left(\mathrm{Ph} \_\mathrm{m}\right)$ en bas de la figure 4 .

Les divergences et convergences d'opérations en «OU » étant peu nombreuses, ce modèle recouvre une combinatoire de 4 gammes, qui sera complétée avec la combinatoire des phases (section suivante). Les quatre gammes sont :

- si le sous-ensemble caisson bas et haut (HCLC) est absent :

- Ph1-Ph4-1, avec une finition peinte,

- Ph1-Ph4-2, avec une finition vernie,

- si le sous-ensemble caisson bas et haut (HCLC) est présent :

- (Ph1etPh2)-Ph3-Ph4-1, avec une finition peinte,

- (Ph1et Ph2)-Ph3-Ph4-2, avec une finition vernie.

La dernière gamme correspond à l'exemple de besoin décrit en fin de section 2.3. Les contraintes entre les ports sont exploitées uniquement en fonction de la présence ou de l'absence du sous-ensemble regroupant les casiers bas et haut, variable HCLC-X. Ces contraintes permettent de sélectionner de manière cohérente la valeur des variables port comportant plusieurs possibilités correspondant aux divergences ou convergences d'opération en « OU » (ports des opérations Op-1 et op-4). 


\subsection{Configuration de phase}

Une phase générique comprend un ou plusieurs ensembles de ressources différentes. Chaque ensemble de ressources est associé à une quantité requise de ressources. En conséquence le sous-modèle associé à la phase est composé de couples de variable (ensemble de ressource, quantités requises) notés (Nom_ens._Ress., Nom_ens._Ress.-Qt). En l'absence de variable quantités requise, la valeur unitaire est considérée par défaut. L'existence du sous-modèle de chaque phase est contrôlée par une contrainte d'activation de sous-modèle reliant la valeur de la variable opération du modèle de la gamme au sous-ensemble correspondant. Les valeurs des variables Nom_ens._Ress. et Nom_ens._Ress.-Qt peuvent être reliées au modèle produit par des contraintes de compatibilité.

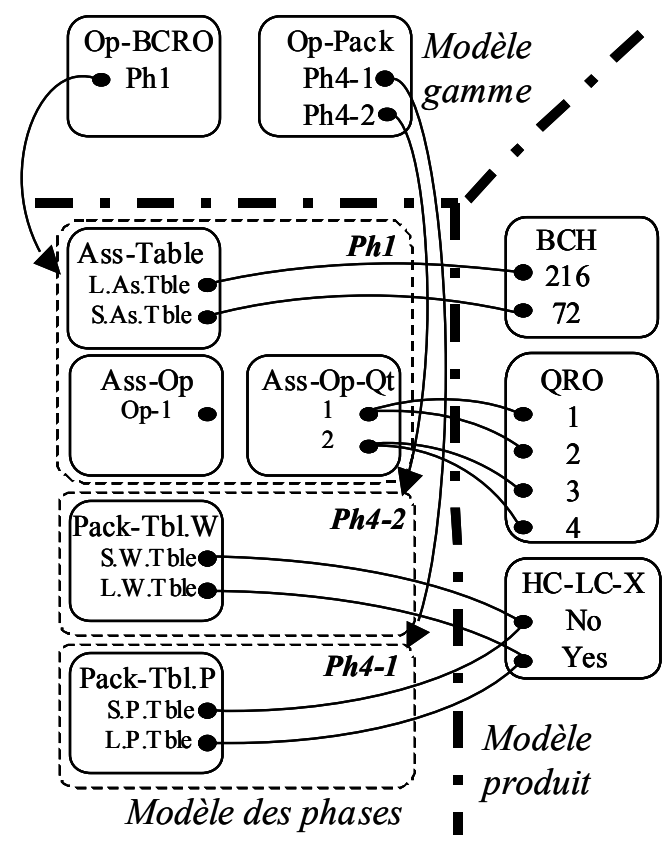

Figure 5. Modèle des phases de l'exemple

A l'aide de ces éléments il est possible de définir le sous-modèle de chaque phase. Dans ce qui suit, seuls les modèles des phases Ph1, Ph4-1 et Ph4-2 sont décrits et représentés en figure 5 :

- la phase Ph1, correspondant à la fabrication du sous-ensemble grand casier et étagères, requiert deux ensembles de ressources différents. Le premier ensemble de ressources table d'assemblage, noté Ass-Table, comprend deux ressources tables : une grande L.As.Tble, et une petite S.AS.Tble devant être utilisées suivant la hauteur du grand casier. Cet ensemble de ressources est requis de manière unitaire. Le 
second ensemble de ressources opérateur, noté Ass-Op, comprend une ressource unique Op-1. La quantité de ressources requises vaut 1 ou 2 suivant le nombre d'étagère du sous-ensemble,

- la phase $\mathrm{Ph} 4-1$, correspondant à l'emballage ou packaging du produit en version peinte, requiert un seul ensemble de ressources. L'ensemble de ressources table pour produits peints, noté Pack-Tbl.P, comprend deux ressources tables : une grande L.P.Tble, et une petite S.P.Tble devant être utilisées suivant la présence du sous-ensemble regroupant les casiers bas et haut. Cet ensemble de ressources est requis de manière unitaire,

- la phase $\mathrm{Ph} 4-2$, correspondant à l'emballage ou packaging du produit en version vernie, requiert un seul ensemble de ressources. L'ensemble de ressources table pour produits vernis, noté Pack-Tbl.W, comprend deux ressources table : une grande L.W.Tble, et une petite S.W.Tble devant être utilisées suivant la présence du sous-ensemble regroupant les casiers bas et haut. Cet ensemble de ressources est requis de manière unitaire,

Chacune de ces phases est reliée au modèle produit par des contraintes de compatibilité :

- phase Ph1 : une contrainte entre les variables (Ass-Table , $\mathrm{BCH}$ ) sélectionnant la table en fonction de la hauteur du grand casier, et une contrainte entre les variables (Ass-Op-Qt , QRO) sélectionnant le nombre d'opérateurs en fonction du nombre d'étagères,

- phase Ph4-1 et Ph4-2: deux contraintes respectivement entre les variables (Pack-Tbl.P , HC-LC-X) et (Pack-Tbl.W , HC-LC-X) sélectionnant la table en fonction de la présence du sous-ensemble HC-LC.

Chaque sous-modèle de phase est relié au modèle gamme par une contrainte d'activation dont la prémisse correspond aux valeurs des variables opération :

- pour la phase $\mathrm{Ph} 1:(\mathrm{Op}-\mathrm{BCRO}=\ll \mathrm{Ph} 1 ») \rightarrow \mathrm{Ph} 1$,

- pour la phase $\mathrm{Ph} 4-1:(\mathrm{Op}-\mathrm{Pack}=\langle\mathrm{Ph} 4-1 ») \rightarrow \mathrm{Ph} 4-1$,

- pour la phase $\mathrm{Ph} 4-2:(\mathrm{Op}-\mathrm{Pack}=« \mathrm{Ph} 4-2 ») \rightarrow \mathrm{Ph} 4-2$.

Sans tenir compte des contraintes du modèle produit, le nombre de phases configurées différentes que l'on peut obtenir à partir de chaque phase générique est de 4 pour la phase $\mathrm{Ph} 1$ et de 2 pour les phases $\mathrm{Ph} 4-1$ et $\mathrm{Ph} 4-2$. Toujours sans tenir compte des contraintes du modèle produit et en considérant que les phases génériques $\mathrm{Ph} 2$ et $\mathrm{Ph} 3$ recouvrent une combinatoire unitaire, le cumul de la diversité des phases et des gammes donne pour chaque gamme identifiée en fin de section 3.2 :

- Ph1-Ph4-1: 4 x $2=8$ gammes,

$-\mathrm{Ph} 1-\mathrm{Ph} 4-2: 4$ × $2=8$ gammes,

$-(\mathrm{Ph} 1$ etPh2)-Ph3-Ph4-1 : (4x1) x 1 x $2=8$ gammes,

- (Ph1et Ph2)-Ph3-Ph4-2 : (4x1) x 1 x $2=8$ gammes, 
Le modèle de la gamme de production générique comprenant gamme générique et phases génériques recouvre donc une combinatoire de 32 gammes de production différentes. Il est à noter que si les phases $\mathrm{Ph} 2$ et $\mathrm{Ph} 3$ recouvraient chacune une combinatoire de deux, la combinatoire s'élèverait à 80 gammes de production différentes montrant ainsi la puissance de représentation de la diversité du modèle proposé.

Lorsque les contraintes du modèle produit sont considérées, cette combinatoire chute car toutes les combinaisons ne sont plus autorisées. Plus précisément, seulement trois instances de la phase $\mathrm{Ph} 1$ ne peuvent exister car il n'est pas possible d'avoir plus de deux étagères avec une hauteur de grand casier de $72 \mathrm{~cm}$. Cela donne suivant les gammes identifiées en fin de section 3.2:

- si le sous-ensemble caisson bas et haut (HCLC) est absent :

- $\mathrm{Ph} 1-\mathrm{Ph} 4-1$, avec une finition peinte $: 3 \times 1=3$ gammes,

- $\mathrm{Ph} 1-\mathrm{Ph} 4-2$, avec une finition vernie : 3 x $1=3$ gammes,

- si le sous-ensemble caisson bas et haut (HCLC) est présent :

- (Ph1etPh2)-Ph3-Ph4-1, avec une finition peinte : $(3 \times 1)$ x 1 x $1=3$ gammes,

- (Ph1etPh2)-Ph3-Ph4-2, avec une finition vernie : $(3 \times 1)$ x 1 x $1=3$ gammes.

Soit 12 gammes de production possibles en respectant le modèle produit recouvrant une diversité de 32 produits configurés différents. La différence entre ces deux nombres est due à ce que la présence ou l'absence du casier haut et les nombres d'étagères (1 ou 2) et (3 et 4) n'ont pas d'impact sur la configuration des gammes de production.

\section{Conclusions}

L'objectif de cette communication était de montrer comment la configuration de produit et celle de sa gamme de production pouvaient être formalisées comme un problème de satisfaction de contraintes.

Dans ce but, la première section a rappelé des éléments de définition de la problématique de configuration de produit et montré comment elle pouvait se mettre sous la forme d'un problème de satisfaction de contraintes. Ce travail a été effectué en utilisant l'extension dynamique des CSP (DCSP) et en la complétant avec un raccourci de notation permettant une modélisation structurée. Le travail s'est principalement intéressé à la représentation du produit configurable avec le formalisme variables/contraintes sans entrer dans l'algorithmique aidant le processus de configuration. Il est important de souligner que tous les algorithmes, supportant le formalisme DCSP, qui permettent de propager ou filtrer les contraintes ou bien de rechercher les solutions peuvent traiter les modèles de configuration de produit proposés. 
La seconde section a étendu les éléments relatifs à la configuration de produit à celle de sa gamme de production. Le modèle proposé met en œuvre les notions de gamme, d'opération, de phase et de ressource. La modélisation de la diversité est obtenue à plusieurs niveaux. Tout d'abord au niveau de la gamme qui est un ensemble d'opérations reliées par des contraintes d'antériorité qui permettent de représenter des réseaux d'opérations avec des convergences et divergences en «ET » et en «OU ». Ces contraintes d'antériorité ont été modélisées avec le concept de port utilisé pour représenter les connexions de composants deux à deux en configuration de produit. A un deuxième niveau, l'opération peut de même représenter une forme de diversité en permettant d'associer plusieurs phases possibles à une même opération. Au dernier niveau la phase est définie comme requérant des ensembles de ressources. Cette notion d'ensemble de ressources autorise de même la représentation d'une autre forme de diversité. Tous les éléments proposés: opérations, antériorités, phases et ressources sont mis en relation avec le modèle produit à l'aide de contraintes qui permettent de configurer la gamme de production en totale cohérence avec le produit configuré.

En ce qui concerne la généricité et les possibilités d'exploitation des éléments proposés, il est à noter qu'il est très difficile de savoir comment fonctionnent les progiciels de configuration du marché. (Sabin el al., 1998) dans leur état du domaine soulignent ce problème. Nous avons eu néanmoins l'occasion dans le cadre du travail effectué avec le groupe Lapeyre de tester les éléments proposés sur des exemples variés avec un progiciel de configuration donné. Les résultats satisfaisants qui ont été obtenus ne permettent cependant pas de conclure que nos propositions sont facilement exploitables avec tous les progiciels de configuration du commerce et toutes les tailles de problèmes. En ce qui concerne la taille et la complexité des problèmes envisageables, il est cependant à noter qu'il a été montré, à la fois sur le plan théorique avec entre autres les notions de diversité compilée (Amilhastre et al., 2002) et sur le plan applicatif (Pargamin, 2002), que les approches par contraintes pouvaient supporter des problèmes de configuration de grande taille et de complexité élevée. Pour terminer, il est également à mentionner que les discussions ou tables rondes informelles tenues avec les éditeurs de progiciels de configuration lors des différents workshop sur la configuration ont clairement montré que la configuration de gamme de production est un problème à considérer.

\section{Bibliographie}

AIEDAM, Special issue on configuration, Artificial Intelligence in Engineering Design and Automation, vol. 12, $\mathrm{n}^{\circ} 4,1998$.

AIEDAM, Special issue on configuration, Artificial Intelligence in Engineering Design and Automation, vol. 17, $\mathrm{n}^{\circ}$ 1, 2003.

Aldanondo M., Fargier H., Véron M., «Configuration, configurateurs et gestion de production », Gestion de Production, traité IC2 , Hermès, 2001, p. 179-209. 
Aldanondo M., Hadj-Hamou K., Moynard G., Lamothe J., "Mass customization and configuration: Requirement analysis and constraint based modeling propositions, Integrated Computer-Aided Engineering, vol. 10, n 2, 2003, p. 177-189.

Amilhastre J., Fargier H., Marquis P., "Consistency Restoration and Explanations in Dynamic CSPs, Application to Configuration , Artificial Intelligence, vol. 135, $\mathrm{n}^{\circ} 1-2,2002$, p. 199-234.

Gelle E., Sabin M., "Solving Methods for Conditional Constraint Satisfaction , Proceedings of IJCAI 2003 Workshop on Configuration, Acapulco, Mexico, 2003, p. 7-12.

Hvam L., Riis J., Malis M., "A multi-perspective approach for the design of configuration systems, Proceedings of ECAI 2002 Workshop on Configuration, Lyon, France, 2002, p. $56-62$.

IEEE, Special issue on configuration, IEEE Intelligent Systems, vol. 13, n 4, 1998.

Mittal S., Falkenhainer B., " Dynamic Constraint Satisfaction Problems », Proceedings of the $9^{\text {th }}$ National Conference on Artificial Intelligence AAAI, Boston, USA, 1990, p. 25-32.

Mittal S., Frayman F., “Towards a generic model of configuration tasks , Proceedings of IJCAI 1989, Detroit, USA, vol. 2, 1989, p. 1395-1401.

Montanari H., "Networks of constraints: Fundamental properties and application to picture processing , Information Sciences, vol. 7, 1974, p. 95-132.

Moynard G., Contribution au déploiement de progiciels de configuration dans l'industrie : éléments de modélisation et d'estimation, Thèse de Doctorat INP de Toulouse 2003.

Pargamin B., "Vehicle Sales Configuration: the Cluster Tree Approach , Proceedings of ECAI 2002 Workshop on Configuration, Lyon, France, 2002, p. 35-40.

Sabin D., Freuder E., "Configuration as Composite Constraint Satisfaction , Proceedings of AAA1 1996 Workshop on configuration, 1996, p. 28-36.

Sabin D., Weigel R., "Product Configuration Frameworks - A Yurvey , IEEE Intelligent Systems, vol. 13, n 4, 1998, p. 42-49.

Soininen T., Gelle E., "Dynamic Constraint Satisfaction in Configuration , Proceedings of AAAI 1999 Workshop on configuration, Orlando, USA, 1999, p. 95-100.

Soininen T., Tiihonen T., Männistö T., Sulonen R., "Towards a General Ontology of Configuration , AIEDAM, vol. 12, n 4, 1998, p. 357-372.

Véron M., Aldanondo M., "Yet another approach to CCSP for configuration problem , Proceedings of ECAI 2000 Workshop on Configuration, Berlin, Germany, 2000, p. 59-62. 